\title{
Original Research \\ Medication discrepancies despite pharmacist led medication reconciliation: the challenges of maintaining an accurate medication list in primary care
}

\author{
Autumn L STEWART, Kevin J LYNCH. \\ Received (first version): 30-Aug-2013 Accepted: 2-Feb-2014
}

\begin{abstract}
${ }^{*}$
Objective: Describe the types of medication discrepancies that persist despite pharmacist-led medication reconciliation using the primary care electronic medical record (EMR).

Methods: Observational case series study of established patients from an urban, indigent care clinic. Medication reconciliation was conducted immediately prior to the physician visit at baseline and return visit. Main outcome measures included: frequency, types, and reasons for discrepancies, patient knowledge, and adherence. Results: There was a $14.5 \%$ reduction in the number of patients with a discrepancy and the frequency of discrepancies was reduced by $7.3 \%$. The rate of medication discrepancies in the chart was reduced by $31.3 \%$. The most common type of discrepancy that persisted at follow up were medications listed on the chart that the patient stopped taking. Discrepancies were more likely to persist in Caucasian subjects when compared to African Americans.

Conclusion: While pharmacist led medication reconciliation appears effective at reducing the likelihood of a medication discrepancy in the EMR, challenges persist in maintaining this accuracy specifically as it relates to patient driven changes to the medication regimen.
\end{abstract}

Keywords: Medication Reconciliation; Electronic Health Records; Medication Errors; Continuity of Patient Care; United States

\section{INTRODUCTION}

Medication reconciliation is, in the shared definition from the American Pharmacists Association and the American Society of Health System Pharmacists, "the comprehensive evaluation of a patient's medication regimen any time there is a change in therapy in an effort to avoid medication errors such as omissions, duplications, dosing errors, or drug interactions, as well as to observe compliance and adherence patterns". ${ }^{1}$ As such, performance of medication reconciliation goes beyond the creation of an accurate medication list. In the Medication Therapy Management in Pharmacy Practice: Core Elements of an MTM Service Model a "Medication

* Autumn L. STEWART. PharmD., BCACP. Assistant Professor of Pharmacy Practice, Division of Clinical, Social and Administrative Sciences. Mylan School of Pharmacy, Duquesne University. Pittsburgh, PA (United States). stewar14@duq.edu

Kevin J. LYNCH. PharmD, BCPS, MBA. Medical

Outcomes Specialist, Pfizer, Inc.

Upper Saint Clair, PA (United States).

kevin.lynch@pfizer.com
Therapy Review" (MTR) is identified as an essential, if not most important step, in the provision of MTM services. ${ }^{2}$

Research in the acute care setting has shown that discrepancies originating in the medication history frequently lead to errors. ${ }^{3}$ The value of the inclusion of pharmacists in medication reconciliation processes in the acute care setting is established. ${ }^{4}$ Error rates, discrepancies, and costs of care are improved when pharmacists lead or participate in an interdisciplinary model for medication reconciliation in the acute setting. ${ }^{5-7}$ While research has demonstrated that the problem of medication record discrepancies are similar in ambulatory care environments, much less is known about the impact of pharmacist-led medication reconciliation on discrepancies in these settings or with the use of an electronic medical record (EMR). ${ }^{8-10}$

A 2009 meta-analysis of studies conducted in primary care demonstrated a lack of quality information on the impact of medication reconciliation. ${ }^{11}$ The studies included in the metaanalysis provided conflicting evidence on the potential for medication reconciliation in ambulatory care to demonstrate a reduction in the rate of discrepancies and the proportion of charts with a discrepancy present. The use of technology has been proposed and explored as a tool for the provision of medication reconciliation that may reduce discrepancies in the medication record. ${ }^{12-14}$ Unfortunately, discrepancies appear to continue at an alarming rate despite the use of an EMR. If "optimizing" medication reconciliation processes through appropriate personnel and technology has an inconsistent impact on discrepancies, then further research is needed to understand where the vulnerabilities continue to exist, such that reconciliation strategies can be further enhanced to manage them.

The objective of this study is to describe the types and causes of medication discrepancies that persist despite pharmacist led medication reconciliation targeted at correcting discrepancies between patient-reported medications and medications listed in the outpatient EMR.

\section{METHODS}

Approval from the University's institutional review board was obtained prior to recruitment of subjects. Indigent, uninsured patients from a free primary 
care center were recruited to participate in the study through a verbal invitation prior to each scheduled physician visit. This verbal invitation occurred immediately following the nurse's intake of the patient. The center, located in an urban setting, provides free medical and dental services to uninsured adults with household incomes of $<200 \%$ of Federal Poverty Limits. Patients eligible for care at the center cannot have private or government health insurance and must be between the ages of 18 and 65 years old. Additional details about the center and the services it provides has been previously published. ${ }^{10}$ Medication reconciliation as a pharmacy led service was established at the site to be conducted by pharmacists and fourth professional year student pharmacists trained and evaluated by the primary investigator. Prior to that time, medication reconciliation was conducted by center nurses and physicians and maintained through the use of an EMR which employs Computerized Physician Order Entry (CPOE) for documentation and generation of prescriptions. Medication lists can be updated automatically through CPOE or manually by any provider at the center before and during implementation of pharmacist led medication reconciliation.

This study included patients from a previous study ${ }^{10}$ for whom there were at least 2 medication reconciliation encounters. Patients were excluded if the first medication reconciliation encounter occurred during their initial appointment to establish care at the center. The decision to use established patients only served as an effective "wash-out period" for patients new to the center whose discrepancies at their initial visit may have been the result of previous care providers elsewhere. Discrepancies present at baseline for established patients reflected the accuracy of a medication list that had been attained through nurse and physician oversight of medication reconciliation, as that was the center's previous method for maintaining the medication list in the EMR prior to the study period. Figure 1 summarizes the recruitment procedures used in this study.

\section{Medication Review}

Medication reviews occurred immediately prior to the patient's visit with the physician. A scannable data collection form was used to provide uniformity and consistency in the documentation of the patient interview; a detailed description of the interview process has been previously published. ${ }^{10}$ Study participants were interviewed to obtain the names, doses, regimens (frequency of administration), and indications of the medications they were currently taking as reported by the patient. The charted medication record was available to assist the pharmacist or student with obtaining a complete and accurate report. Outside physicians or patients' community pharmacies could be contacted to obtain additional information if needed, although done infrequently. When available, prescription drug bottles were reviewed to ensure the accuracy of the patient report. As part of the medication review, a

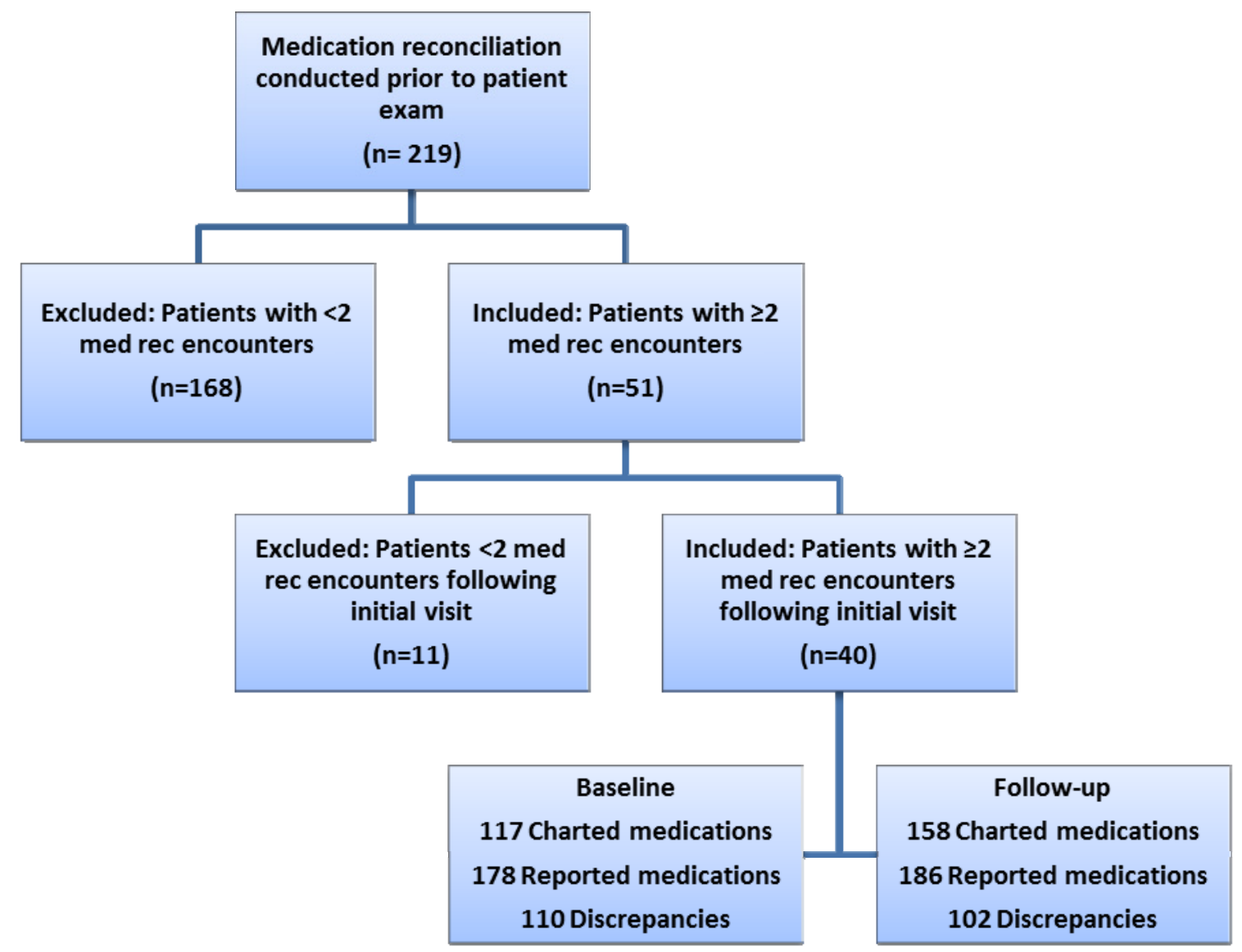

Figure 1. Study design and subject recruitment 
comparison between the patient's "Reported Medications" and "Charted Medications" from the EMR was made. Any discrepancy found was further categorized by "Discrepancy Type" and "Reason for Discrepancy". Discrepancies were verbally conveyed and discussed with the physician in addition to any other medication related problems identified during the review. The medication list in the EMR was subsequently corrected by the pharmacist or student conducting the review and updated following the physician visit if the provider verbally communicated any changes to the pharmacist or student pharmacist.

Demographics including: age, gender, and ethnicity were also collected. The authors anticipated the interview itself could potentially serve as an intervention that would affect patient adherence and/or patient medication knowledge through a Hawthorne effect. A change in medication adherence (positively or negatively) between baseline and follow up could lead to discrepancies in the EMR. To account for this potential confounder, subjects were also screened for medication adherence using the Morisky scale, a validated, 4-item questionnaire which utilizes selfreport to identify patients who may exhibit nonadherent medication taking behaviors to maintenance medications. ${ }^{15}$ The Morisky scale provided an "adherence score" for each patient ranging $0-4$, with $0-1$ indicating low level of adherent behavior, 2-3 indicating medium level of adherent behavior, and 4 being high adherence. Patient medication knowledge was seen as another potential confounder; interviewers also assessed patient knowledge of medication name, dose, regimen, and indication. To achieve an objective and systematic method for assessment of patient knowledge, the number of medications for which the patient was able to accurately recall the drug name, dose, regimen, and indication was compared proportionally to the total number of reported medications. The thresholds of $0 \%, 25 \%, 50 \%$, $75 \%$, and $100 \%$ were selected arbitrarily for convenience and standardization (e.g. a patient knowing the dose of 4 of the 8 medications being taken would receive a knowledge score of $50 \%$ for that parameter).

\section{Data Analysis}

All data were entered from the scannable data collection form directly into Microsoft Access 2007 (Microsoft Corp. Redmond, Wa) database and were analyzed using IBM SPSS Statistics for Windows ${ }^{\circledR}$ (IBM Corp. Version 20.0. Armonk, NY) and Minitab version 15.1 (Minitab, State College, PA). Descriptive statistics were utilized for all parameters and were reported as mean and SD or range for continuous variables, and count and $/$ or percentages (\%) for dichotomous variables. Inferential statistics were conducted using Paired-t test for continuous variables, McNemar's test for ordinal data, and Chi-Squared and Fisher's exact test for dichotomous variables. A patient specific discrepancy rate was calculated for each visit by dividing the number of discrepancies present by the number of medications listed on the chart. A categorical variable for comparisons among age, gender, and ethnicity was created based on discrepancy status at follow-up. Subjects with no change in or increased discrepancies were categorized as "Discrepancies Persisted"; those with a reduction in the number of discrepancies were labeled "Discrepancies Resolved". Results were considered statistically significant if the $p$ value was <0.05. Sample size determination was completed using Minitab Software. For $80 \%$ power and a population of approximately 2000 patients a sample size of 42 was needed (assuming a standard deviation of 15 and an alpha level of 0.05 ).

\section{RESULTS}

Medication reconciliation was conducted in 219 patients over a time period of 13 months. Of these patients, 51 met the inclusion criteria by having multiple medication reconciliation encounters. Eleven of the remaining 51 patients were excluded from data analysis because the initial medication reconciliation encounter was their initial visit at the center. There were 40 patients eligible to participate in the study of which $23(58 \%)$ were female. Nineteen patients $(47.5 \%)$ were African American and 19 were Caucasian. The remaining 2 patients were Latino or Other. Patients ranged in age 23 to 64 years old, with a mean of 49 years of age. The mean time between the baseline and follow-up encounter was 121 days. The mean number of medications reported by the patient was not changed between baseline and follow-up, 4.5 (range 1-9) and 4.7 (range 0-11) respectively. The mean number of medications listed in the chart increased from 2.9 (range 1-9) at baseline to 4 (range 1-11) at follow up.

\section{Medication Discrepancies}

The presence of a discrepancy at baseline was identified in $39(97.5 \%)$ patients. At the follow up encounter, discrepancies remained in 33 (82.5\%) patients demonstrating a $14.5 \%$ reduction in the number of patients with a discrepancy present. A breakdown of discrepancy rates for each type of medication discrepancy at baseline and follow-up is depicted in Table 1. Because the number of medications on the chart and reported by the patient were different between baseline and follow-up, the occurrence of a discrepancy must be interpreted in light of the whole number of medications from each source at that time. At baseline the discrepancy rate to the number of charted medications was $110 / 117$. The rate of discrepancy to number of medications reported by the patient was $110 / 178$. At follow-up there were 102 discrepancies present to 158 charted medications and 186 patient reported medications. This represents an overall $31.3 \%$ reduction in the discrepancy rate based on the number of charted medications and an $11.3 \%$ reduction in the rate of discrepancies compared to the number of reported medications. The mean patient specific discrepancy rate at baseline (0.994) was not significantly different from the mean discrepancy rate at follow-up $(0.715, p=0.255)$. 


\begin{tabular}{|c|c|c|}
\hline Discrepancy Type & $\begin{array}{l}\text { Frequency, } \\
\text { No. }(\%) \\
\text { at Baseline* }\end{array}$ & $\begin{array}{c}\text { Frequency, } \\
\text { No.(\%) } \\
\text { at Follow-up† }\end{array}$ \\
\hline $\begin{array}{r}\text { Medication on Chart } \\
\text { Discrepancy with a medication listed in the chart } \\
\text { Patient Did Not Report } \\
\text { Medication with Automatic Stop Date } \\
\text { Patient Stopped Medication } \\
\text { Change during recent office visit } \\
\text { Discontinued medication during recent office visit } \\
\text { Other types of reasons }\end{array}$ & $\begin{array}{r}42(38.2) \\
26(61.9) \\
0(0) \\
5(11.9) \\
6(14.3) \\
1(2.4) \\
4(9.5)\end{array}$ & $\begin{array}{r}47(46.1) \\
14(29.8) \\
9(19.1) \\
11(23.4) \\
5(10.6) \\
6(12.8) \\
2(4.3)\end{array}$ \\
\hline $\begin{array}{l}\text { Medication omitted on chart } \\
\text { Discrepancy with a reported medication not on the chart } \\
\qquad \begin{array}{r}\text { Outside MD prescribed without communiqué } \\
\text { Patient did not report medication correctly } \\
\text { Other types of reasons }\end{array}\end{array}$ & $\begin{array}{r}44(40) \\
32(72.3) \\
7(15.9) \\
0(0) \\
5(11.4) \\
\end{array}$ & $\begin{array}{r}40(39.2) \\
29(72.5) \\
4(10.0) \\
3(7.5) \\
4(10) \\
\end{array}$ \\
\hline $\begin{array}{l}\text { Different Dose } \\
\text { Discrepancy between dose reported and dose charted } \\
\text { Patient did not report medication correctly } \\
\text { Change during recent office visit } \\
\text { Outside MD prescribed without communiqué }\end{array}$ & $\begin{array}{l}7(70) \\
1(10) \\
2(20) \\
\end{array}$ & $\begin{array}{r}6(5.9) \\
6(100) \\
0(0) \\
0(0)\end{array}$ \\
\hline $\begin{array}{l}\text { Different Regimen } \\
\text { Discrepancy between regimen reported and regimen charted } \\
\text { Patient Changed Medication } \\
\qquad \begin{array}{r}\text { Patient did not report medication correctly } \\
\text { Other types of reasons }\end{array}\end{array}$ & $\begin{array}{r}12(10.9) \\
8(66.7) \\
0(0) \\
4(33.3) \\
\end{array}$ & $\begin{array}{rr}7(6.9) & \\
0(0) \\
1(14.3) \\
6(85.7) \\
\end{array}$ \\
\hline
\end{tabular}

Of the 110 discrepancies identified at baseline, the most common type (40\% of discrepancies) was a patient reported medication that was omitted from the chart. While the frequency of this discrepancy was not changed at follow-up (39.2\%), the most common type of discrepancy at follow up shifted to a medication listed on the chart. Despite the increase in frequency, there was a $46.2 \%$ reduction in the presence of a discrepancy listed on the chart that was caused by the patient's failure to report the medication. The increase in this category type was primarily caused by medications with a finite time frame for use and discontinuations made by the provider or patient. The most common discrepancy types and reasons are depicted in Table 1. The types of discrepancies were further characterized by reasons for the discrepancy as depicted in Table 2.

Neither age nor gender were associated with the persistence of discrepancies. However, an association was observed in the persistence of discrepancies at follow up among Caucasian subjects when compared to African Americans $(p=0.047)$ despite a lower median number of discrepancies at baseline (2 and 2.5, respectively).

\section{Medication Adherence and Knowledge}

The median adherence score at baseline was 2 , at follow up this had not changed. At baseline, less than half of patients $(47.5 \%)$ were $>75 \%$ knowledgeable on all medication parameters (name, dose, regimen, indication). At follow-up this number had improved to $57.5 \%$, however the difference failed to reach statistical significance.

\section{DISCUSSION}

\section{Discrepancies}

This study demonstrates the elusive nature of reducing discrepancies and inaccuracies in the EMR over time, which adds to previous research on the issue. ${ }^{7,8,12}$ While the discrepancy rate for individual patients was not changed, the number of patients with a discrepancy present was reduced, and the overall presence of discrepancies across all patients was reduced by $31.3 \%$, representing an improvement to a more accurate medication record. Recall in this study, that the discrepancies present at baseline were those identified during the initial pharmacist encounter during transition from "nurse and physician led" to "pharmacist led" medication reconciliation. The discrepancy types present at follow up were those that persisted following a previous medication reconciliation encounter led by a pharmacist. The increase in the number of medications listed on the chart between baseline and follow-up also suggest that a more complete picture of the patient's medication record was available at follow-up. This may be the result of intentional time and process dedicated to medication reconciliation itself but is also likely to be contributed to by the knowledge of prescription and non-prescription medication unique to pharmacists. While the goal of reduced discrepancies may be a sliding target because discrepancies may persist, the medication list overall may have been made more accurate for future visits. While processes and personnel dedicated to medication reconciliation may improve the accuracy of a medication list created at a given point of time, it does not represent an accurate estimation of what occurs based on patient behavior outside of those assessments. Overall, there was a movement towards a medication list with fewer discrepancies; 


\begin{tabular}{|l|c|c|}
\hline \multicolumn{1}{|c|}{ Table 2. Overall discrepancy reasons, independent of type at baseline and follow-up } \\
\hline \multicolumn{1}{|c|}{ Discrepancy } & $\begin{array}{c}\text { Frequency, No. (\%) } \\
\text { at baseline N=92 }\end{array}$ & $\begin{array}{c}\text { Frequency, No.(\%) } \\
\text { at follow-up N=95 }\end{array}$ \\
\hline OTC use & $33(35.9)$ & $31(32.6)$ \\
\hline Patient did not report & $22(23.9)$ & $24(25.3)$ \\
\hline Outside physician prescribed without communiqué & $14(15.2)$ & $5(5.3)$ \\
\hline Patient changed/stopped medication & $14(15.2)$ & $11(11.6)$ \\
\hline Medication with automatic stop date & $0(0)$ & $7(7.4)$ \\
\hline Change during recent office visit & $6(6.5)$ & $6(6.3)$ \\
\hline Stopped during recent office visit & $0(0)$ & $6(6.3)$ \\
\hline Initiation of new medication, recent office visit & $3(3.3)$ & $2(2.1)$ \\
\hline Other & $0(0)$ & $3(3.2)$ \\
\hline
\end{tabular}

however some discrepancy types persisted (omissions) and others increased (medications listed in the EMR).

Previous research has shown that discrepancies with medications listed in the EMR are a common problem. $^{16}$ The current study shows this type of discrepancy is likely to persist despite a standardized method for medication reconciliation led by a pharmacist. Vulnerabilities in other steps of the process exist which may contribute to discrepancies; these steps are opportunities for improvement to "optimize" the full process. The clinical significance of this type of discrepancy occurs during the clinical decision making where the prescriber may assume the presence of a therapy that either the patient stopped taking on their own or may have been discontinued by another provider, leading to confusion when transitions of care occur. The reason for the discontinuation either by patient or provider is unclear whether intended or not. In this study, while an increase in the frequency of discrepancies with a medication listed in the chart was observed and is a perceived negative effect, it could represent a beneficial impact depending on the reason for the discrepancy. For example, in this category, there was a reduction in a discrepancy that was caused by the patient's failure to report which suggests that medication reconciliation successfully elicited a complete medication list at the previous appointment. The subsequent causes of discrepancies where there was an increase were related to automatic stop dates or changes made by the patient or a prescriber since the last appointment. If that is the case, then the benefit of a more accurate list outweighs the risk of an inaccuracy at a later date. The large increase in discrepancies in medications with an automatic stop date at follow-up also support the finding that the pharmacist at the previous encounter had improved documentation of short term therapies initiated at that time. While this may have led to an increase in discrepancies, it does suggest that the medication list overall became more accurate and supports conducting medication reconciliation at every visit. Documentation of discontinued therapies may be clinically relevant for adverse drug reaction monitoring and future drug selection. The responsibility of documenting physician led changes to or discontinuations of medications during or between physician visits is shared among all with access to the medication list. An EMR or CPOE system allowing the entry of anticipated or automatic stop dates upon prescribing could also be beneficial; however, this could also lead to medications being prematurely removed from the medication list prior to the patient's use or discontinuation of them which would further complicate the accuracy of a subsequent medication list.

The discrepancies with medications not listed in the chart continued to be primarily related to use of OTC medications. These findings emphasize the need for medication reconciliation to be conducted at each interface of care where changes are made and to engage the patient in making regular updates to their own medication list. While the use of OTC drugs is by definition, safe and effective without a physician's oversight, the potential for drug interactions, therapeutic duplications, and adverse effects associated with OTCs (NSAIDs, decongestants, etc.) does exist. Anecdotally, one patient did indicate on a follow up encounter that upon returning home following the previous visit that she had intentionally reviewed the names and doses of her medications to be more prepared for future visits. Despite patients being "trained" to report their medications at each visit, patients not reporting medications continued to be the reason behind approximately a quarter of the discrepancies occurring. The role of the patient, and his or her medication taking behaviors, cannot be ignored as a contributing factor that drives the presence of discrepancies. Providers must assume that the value in a medication list's accuracy depreciates as soon as the patient leaves the office. Strategies for medication reconciliation that engage the patient or employ a more patient centered approach may have benefit in reducing this category of discrepancies.

\section{Patient Characteristics}

An unexpected and interesting finding in the present study was the statistically significant association with the persistence of discrepancies among Caucasian subjects despite fewer discrepancies at baseline. While it is unclear from this study what contributed to this apparent disparity, it does generate interest in further exploration of ethnic differences and optimization of medication reconciliation processes. It is possible that factors such as patient-provider relationship and trust could have contributed to these findings. Research conducted by Johnson and colleagues found that physicians demonstrated a more verbally dominant and less patient centered communication style with African American patients. ${ }^{17}$ In medication reconciliation, the opportunity for patient report is essential to garner an accurate list; if communication is primarily provider led, then the ability to effectively do this is limited. The intentional time and opportunity dedicated to medication 
reconciliation in this study might have overcome this potential barrier by providing the patient time to discuss medications, independent of the provider's communication techniques. While this may explain the improvement seen among African American subjects, it does not explain the persistence of discrepancies among Caucasians. Program evaluations and future studies on medication reconciliation processes should take into account this disparity and consider opportunities for improvement.

Medication adherence did not change from baseline to follow up. While the authors anticipated a change in patient medication knowledge from baseline, the $21 \%$ improvement seen did not reach statistical significance. While no difference as a result of the intervention may be possible, it may also be a result of the small sample size and being underpowered to detect significance. This expectation for improvement was based on anecdotal reports of patients at follow-up stating they had looked up the name and dose of their medication as a result of being asked this information at their previous appointment. This does suggest that patients became more "engaged" in their medications as a result of the dialogue occurring with medication reconciliation. Further research is needed to determine if conversation around medications during reconciliation has a downstream effect on other outcomes such as patient-provider relationships, medication literacy, and other subtleties with health behavior change.

Additional research is also needed to explore what interventions can be effective in reducing specific types of discrepancies. For example, online patient medication record programs that interface with the prescribers' EMR and allow patients to record medication changes or use of self-care therapies, could have an impact on the rates and types of discrepancies discussed previously.

\section{Limitations}

The present study is not without limitations with respect to the general findings. The small sample size prohibited statistically significant changes to be observed. The small sample size from the data collection period was likely due to challenges specific with the recruitment procedures and inherent to the population of patients being studied. The invitation to participate in the study was conducted at each encounter to maintain blinding of the interviewer from knowledge of which patients had previous encounters present. This blinding prevented the introduction of bias which could have resulted from interviewers' differing approaches to patients upon initial versus follow-up encounter. While this method of recruitment prevented interviewer bias, it did lead to challenges in recruiting a large sample of patients with multiple encounters present. It was unknown throughout the data collection period how many patients were seen on multiple occasions as the unblinding occurred in the data analysis phase. A study population of uninsured, low income adults presents unique challenges with large numbers of patients being lost to follow-up as a result of not having subsequent visits captured. It was important though to only include patients established at the center so as to not count discrepancies that would have naturally existed prior to establishing care at this center. While the provision of medication reconciliation services was continued unchanged, the intensive nature of the study data collection and an increasing volume of patients led to a curtailed data collection beyond the study period which resulted in smaller than ideal sample numbers. Another limitation was that the clinical importance of the discrepancies identified was not described beyond the "discrepancy reason". This limits our ability to accurately predict the discrepancies' impact on patient outcomes, including relative risk of harm to the patient. Another limitation was the use of convenience sampling; an uninsured, indigent population may not be representative of patients across all primary care centers due to lower socioeconomic status and disparities in disease prevalence. Despite measures to improve inter-rater reliability through training and a standard data collection form, differences in documentation and judgment could have existed and resulted in variations between interviewers. Inter-rater reliability was not measured; thus it is not possible to discern to what extent this limitation affected the outcomes.

\section{CONCLUSIONS}

Medication reconciliation processes remain an important initiative for identifying discrepancies between the medication list in the EMR and patients' actual medication taking behaviors. Pharmacist led medication reconciliation in an ambulatory, indigent care clinic produced an overall more complete list of medications, however, discrepancies remained frequent and were usually related to patient or physician changes since the last appointment or medications with automatic stop dates. Further research on patient adherence and engagement is needed to identify effective interventions that reduce these types of persistent discrepancies.

\section{CONFLICT OF INTEREST}

Dr. Stewart has no conflicts of interest to disclose. Dr. Lynch is an employee of Pfizer, Inc.

Portions of this work have been presented in abstract and poster form at APhA 2012 Annual Meeting.

\section{DISCREPANCIAS DE LA MEDICACIÓN A PESAR DE LA RECONCILIACIÓN DE LA MEDICACIÓN POR FARMACÉUTICO: EL RETO DE MANTENER UNA LISTA DE MEDICACIÓN PRECISA EN ATENCIÓN PRIMARIA}

\section{RESUMEN}

Objetivo: Describir los tipos de discrepancias que persisten a pesar de una reconciliación de la medicación hecha por farmacéutico usando la historia clínica electrónica (EMR) de atención primaria.

Métodos: Estudio observacional de serie de casos en una clínica urbana de atención a indigentes. Se realizó 
reconciliación de la medicación inmediatamente antes de la consulta médica al inicio y en la visita de continuación. Los principales resultados medidos incluían: frecuencia, tipos y motivos de las discrepancias, conocimiento del paciente y cumplimiento.

Resultados: Hubo una reducción del 14,5\% en el número de pacientes con discrepancias y la frecuencia de discrepancias se redujo en un 7,3\%. La tasa de discrepancias de medicación en la historia se redujo en un $31,3 \%$. Los tipos más frecuentes de discrepancias que persistían en el seguimiento fueron medicaciones listadas en el historial que el paciente había dejado de tomar. Las discrepancias eran más frecuentes en individuos caucásicos que en afro-americanos.

Concusión: Aunque la reconciliación de la medicación hecha por farmacéuticos parece ser efectiva reduciendo la probabilidad de discrepancias en la medicación de la EMR, existen retos para mantener la precisión, especialmente en lo que se refiere a los cambios del régimen de medicación realizados por el paciente.

Palabras clave: Reconciliación de la Medicación; Registros electrónicos de Salud; Errores de Medicación; Continuidad de los Cuidados al Paciente; Estados Unidos

\section{References}

1. Chen D, Burns A. Summary and Recommendations of ASHP-APhA Medication Reconciliation Initiative Workgroup Meeting, February 12, 2007. Available at: http://www.ashp.org/s_ashp/docs/files/MedRec_ASHP_APhA_ Wkgrp_MtgSummary.pdf (Accessed June 6, 2012).

2. American Pharmacists Association, National Association of Chain Drug Stores Foundation. Medication therapy management in pharmacy practice: core elements of an MTM service model (version 2.0). http://www.pharmacist.com/AM/Template.cfm?Section=Home2\&CONTENTID=15496\&TEMPLATE=/CM/ContentDisplay .cfm (Accessed March 3, 2010).

3. Gleason KM, McDaniel MR, Feinglass J, Baker DW, Lindquist L, Liss D, Noskin GA. Results of the Medications At Transitions and Clinical Handoffs (MATCH) study: an analysis of medication reconciliation errors and risk factors at hospital admission. J Gen Intern Med. 2010;25(5):441-447. doi: 10.1007/s11606-010-1256-6

4. Ketchum K, Grass CA, Padwojski A. Medication reconciliation: verifying medication orders and clarifying discrepancies should be standard practice. Am J Nurs. 2005;105(11):78-85.

5. Nester TM, Hale LS. Effectiveness of a pharmacist-acquired medication history in promoting patient safety. Am J Health Syst Pharm. 2002;59(22):2221-2225.

6. Gleason KM, Groszek JM, Sullivan C, Rooney D, Barnard C, Noskin GA. Reconciliation of discrepancies in medication histories and admission orders of newly hospitalized patients. Am J HealthSyst Pharm. 2004;61(16):1689-1695.

7. Varkey P, Cunningham J, O'Meara J, Bonacci R, Desai N, Sheeler R. Multidisciplinary approach to inpatient medication reconciliation in an academic setting. Am J Health Syst Pharm. 2007;64(8):850-854.

8. Bedell SE, Jabbour S, Goldberg R, Glaser H, Gobble S, Young-Xu Y, Graboys TB, Ravid S. Discrepancies in the use of medications. Arch Intern Med. 2000;160(14):2129-2134.

9. Johnson CM, Marcy TR, Harrison DL, Young RE, Stevens EL, Shadid J. Medication reconciliation in a community pharmacy setting. J Am Pharm Assoc (2003). 2010;50(4):523-526. doi: 10.1331/JAPhA.2010.09121

10. Stewart AL, Lynch KJ. Identifying discrepancies in electronic medical records through pharmacist medication reconciliation. J Am Pharm Assoc (2003). 2012;52(1):59-66. doi: 10.1331/JAPhA.2012.10123

11. Bayoumi I, Howard M, Holbrook AM, Schabort I. Interventions to Improve Medication Reconciliation in Primary Care. Ann Pharmacother. 2009;43(10):1667-1675. doi: 10.1345/aph.1M059

12. Peyton L, Ramser K, Hamann G, Patel D, Kuhl D, Sprabery L, Steinhauer B. Evaluation of medication reconciliation in an ambulatory setting before and after pharmacist intervention. J Am Pharm Assoc (2003). 2010;50(4):490-495. doi: 10.1331/JAPhA.2010.09055

13. Bassi J, Lau F, Bardal S. Use of information technology in medication reconciliation: a scoping review. Ann Pharmacother. 2010;44(5):885-897. doi: 10.1345/aph.1M699

14. Nemeth LS, Wessell AM. Improving Medication Safety in Primary Care Using Electronic Health Records. J Patient Saf. 2010;6(4):238-243.

15. Morisky DE, Green LW, Levine DM. Concurrent and Predictive Validity of a Self-Reported Measure of Medication Adherence. Med Care. 1986;24(1):67-74.

16. Orrico K. Sources and types of discrepancies between electronic medical records and actual outpatient medication use. J Manag Care Pharm. 2008;14(7):626-631.

17. Johnson RL, Roter D, Powe NR, Cooper LA. Patient Race/Ethnicity and Quality of Patient-Physician Communication During Medical Visits. Am J Public Health. 2004;94(12):2084-2090. 